\title{
QUEIXA DE VERTIGEM E HIPERTENSÃO ARTERIAL
}

\author{
Vertigo complaint and blood hypertension
}

\author{
Luciana Lozza de Moraes Marchiori ${ }^{(1)}$, Eduardo de Almeida Rego Filho ${ }^{(2)}$
}

\begin{abstract}
RESUMO
Objetivo: investigar a presença de queixa de vertigem em pacientes de meia idade com hipertensão arterial. Métodos: estudo do tipo prospectivo, transversal. Composto por 154 indivíduos de ambos os gêneros com idade de 45 a 64 anos. A hipertensão foi verificada por meio de medição da pressão arterial e de questionário sistematizado sobre hipertensão e uso de medicamentos para pressão arterial. A queixa de vertigem foi verificada por meio de anamnese audiológica. Resultados: pode-se verificar que existe associação significante entre hipertensão arterial e queixa de vertigem. Conclusão: os resultados da presente pesquisa, por meio da constatação da associação entre hipertensão arterial e queixa de vertigem, servirão de base a profissionais da área de saúde que estão envolvidos com sintomas provenientes da hipertensão arterial.
\end{abstract}

DESCRITORES: Hipertensão; Vertigem; Tontura; Qualidade de Vida; Meia-Idade

\section{INTRODUÇÃO}

A tontura é a ilusão de movimento do próprio indivíduo ou do ambiente que o circunda. Este sintoma pode ser causado por uma disfunção em qualquer segmento dos sistemas relacionados ao equilíbrio corporal. Quando a tontura, que é considerada como um dos sintomas mais comuns entre adultos, adquire caráter rotatório é denominada vertigem ${ }^{1,2}$.

A elevada incidência de distúrbios labirínticos se deve em grande parte à hipersensibilidade do labirinto a vários distúrbios como, por exemplo: hormonais, metabólicos, cervicais e circulatórios. Estudos mostram que alterações auditivas e vestibulares podem ser secundárias à hipertensão arterial sistêmica ${ }^{3,4}$. Nas vestibulopatias de origem vascular, pode-se observar sintomas otoneurológicos leves, moderados ou graves, e a crise labiríntica pode preceder o comprometimento cardíaco ${ }^{4}$.

Todas as células vivas precisam de fornecimento adequado de oxigênio e nutrientes para manterem suas funções, e tal fornecimento depende da integridade funcional e estrutural do coração e dos vasos sangüíneos ${ }^{5}$. A hipertensão arterial pode

(1) Fonoaudióloga; Professora no Curso de Fonoaudiologia e Mestrado em Odontologia da Universidade Norte do Paraná; Doutora em Medicina e Ciências da Saúde pela Universidade Estadual de Londrina - PR

(2) Médico Nefrologista; Docente no Programa de Pós-graduação em Medicina e Ciências da Saúde da Universidade Estadual de Londrina; Doutor em Medicina pela Universidade Estadual de Londrina - PR facilitar alterações estruturais do coração e vasos sanguíneos ${ }^{6}$.

O comprometimento do aparelho circulatório pode prejudicar o ouvido interno sendo que, dos mecanismos fisiopatológicos descritos é o aumento da viscosidade sanguínea, que acarreta uma diminuição do fluxo sanguíneo capilar e o transporte de oxigênio ${ }^{7}$.

A patologia do aparelho circulatório pode afetar diretamente o ouvido interno de muitas maneiras. Um dos mecanismos fisiopatológicos vasculares descritos é o aumento da viscosidade sanguínea, a qual acarreta uma diminuição do fluxo sanguíneo capilar que acaba por diminuir o transporte de oxigênio levando a hipóxia tecidual ${ }^{6-8}$.

O objetivo deste trabalho foi verificar a freqüência da queixa de vertigem em pacientes com e sem hipertensão arterial com idade entre 45 a 64 anos sem perda auditiva analisando a possível associação entre queixa de vertigem e hipertensão arterial.

\section{MÉTODOS}

Foram incluídos 154 indivíduos entre 45 e 64 anos, sem perda auditiva constatada por meio de audiometria tonal liminar. A população foi selecionada na seqüência dos atendimentos da Clínica de Fonoaudiologia da Universidade Norte do Paraná. Foram excluídos pacientes com história prévia de traumatismo craniano, diabetes, acidente vascular cerebral, portadores de nefropatia, com história de internações ou uso de medicamentos com drogas potencialmente ototóxicas. 
Todos os pacientes foram entrevistados pela fonoaudióloga responsável pela pesquisa juntamente com bolsistas de iniciação científica da citada universidade. Foi aplicado um questionário para preenchimento sistematizado de dados clínicos tais como nome, idade, gênero e escolaridade e as seguintes perguntas: o senhor tem pressão alta? tem medido sua pressão arterial ultimamente? quando mediu a pressão pela última vez? quanto tem dado sua pressão arterial? o nome do médico? ou a Unidade de Saúde onde faz controle? toma remédio para a pressão arterial, qual ou quais os medicamentos que toma ${ }^{8}$. A pressão arterial foi tomada por meio de método indireto, auscultatório com manguito de tamanho adequado e manômetro de coluna de mercúrio da marca Tycos $\AA$. Foram respeitadas as normas estabelecidas pelo III Consenso Brasileiro de Hipertensão Arterial de $1998^{9,10}$. Sendo considerados portadores de hipertensão arterial àqueles com pressão arterial $=140$ por $90 \mathrm{~mm}$ de $\mathrm{Hg}$ em várias tomadas e ou àqueles que responderam afirmativamente as perguntas 1 e 6 e identificaram o(s) medicamento(s) que tomavam.

A aferição da presença da variável queixa de vertigem foi realizada baseada no protocolo para anamnese audiológica ${ }^{5}$ utilizada na rotina de atendimentos, onde verificou-se se cada paciente já apresentou alguma crise vertiginosa ou se apresenta atualmente a citada queixa.

A pesquisa foi realizada através de estudo prospectivo, transversal. O projeto foi analisado e aprovado pela Comissão de ética da instituição com o número 082/06.

A associação entre a queixa de vertigem e hipertensão arterial foi analisada mediante as estimativas da odds ratios (OR), por ponto e por intervalo e do valor da estatística pelo Teste Qui-quadrado de Mantel-Haenszel e Teste Exato de Fischer.

\section{RESULTADOS}

Pode-se observar que a hipertensão arterial apresentou associação significante $(p<0,05)$ com a queixa de vertigem (Tabela1).

Houve uma proporção maior de mulheres em ambos os grupos, sendo 119 (77,3\%) da população do gênero feminino e $35(22,7 \%)$ do gênero masculino. Quanto à idade, houve uma média de idade de $55 \pm$ 5,1 no grupo com hipertensão arterial e de $51,8 \pm 5,1$ no grupo sem hipertensão arterial (Tabelas 2 e 3 ).

Em relação à escolaridade, ambos os grupos apresentaram maior freqüência de indivíduos com primeiro grau completo. Entre os que apresentavam hipertensão arterial, $42(91,3 \%)$ não eram fumantes e entre os sem hipertensão arterial 90 (83,3\%) não eram fumantes.

Quanto ao uso de fármacos anti-hipertensivos, constatou-se que dos 46 indivíduos que tinham hipertensão, apenas um $(2,1 \%)$ não fazia uso de medicamentos. Quanto à associação de fármacos antihipertensivos, 11 indivíduos (23,9\%), faziam uso de mais de um fármaco anti-hipertensivo. Prevalecendo a associação de diuréticos mais inibidores de ECA (enzima conversora de angiotensina) em cinco indivíduos $(10,8 \%)$ e o uso de inibidores de ECA em 17 $(36,9 \%)$ dos sujeitos. Além de, $14(30,4 \%)$ fazerem uso de diuréticos, $15(32,6 \%)$ fazerem uso de â bloqueadores, três $(6,5 \%)$ fazerem uso de inibidores de antagonista de angiotensina II, dois (4,3\%) fazerem uso de bloqueadores de canal de Cálcio, um (2,1\%) fazer uso de á - bloqueadores e cinco (10,9\%) fazerem uso de outros anti-hipertensivos tais como, cloridina, á - metildopa e minoxidil. Além de quatro $(8,7 \%)$ dos pacientes estarem fazendo uso de outros fármacos concomitantemente, tais como, antiarrítmicos, vasodiltadores, e antiagregantes plaquetários (Tabela 4).

Tabela 1 - Distribuição completa do número de pacientes segundo hipertensão arterial e queixa de vertigem

$$
\text { Vertigem }
$$

\begin{tabular}{|c|c|c|c|c|c|}
\hline \multirow{2}{*}{$\begin{array}{l}\text { Hipertensão } \\
\text { Arterial }\end{array}$} & \multicolumn{2}{|c|}{ Sim } & \multicolumn{2}{|c|}{ Não } & \multirow[t]{2}{*}{ TOTAL } \\
\hline & $\mathrm{n}$ & $\%$ & $\mathrm{n}$ & $\%$ & \\
\hline Sim & 13 & 28,26 & 33 & 71,74 & 46 \\
\hline Não & 12 & 11,11 & 96 & 88,89 & 108 \\
\hline TOTAL & 25 & & 129 & & 154 \\
\hline
\end{tabular}

$\mathrm{OR}=3,1515[1,3089$, OR $<7,5879] ; \div_{\text {corr }}^{2}=5,773(p=0,0163)$

n- número absoluto 
Tabela 2 - Distribuição dos pacientes do gênero feminino com e sem queixa de vertigem em relação à hipertensão arterial

\begin{tabular}{|c|c|c|c|c|c|}
\hline \multicolumn{6}{|c|}{ Vertigem (Gênero Feminino) } \\
\hline \multirow{2}{*}{ Arterial } & & & & & TOTAL \\
\hline & $n$ & $\%$ & $\mathrm{n}$ & $\%$ & \\
\hline Sim & 8 & 22,86 & 27 & 77,14 & 35 \\
\hline Não & 11 & 13,10 & 73 & 86,90 & 84 \\
\hline TOTAL & 19 & & 100 & & 119 \\
\hline
\end{tabular}

Tabela 3 - Apresenta a distribuição dos pacientes do gênero masculino com e sem queixa de vertigem em relação à hipertensão arterial

\begin{tabular}{|c|c|c|c|c|c|}
\hline \multirow{2}{*}{ Arterial } & \multicolumn{2}{|c|}{ Sim } & \multicolumn{2}{|c|}{ Não } & \multirow[t]{2}{*}{ TOTAL } \\
\hline & $n$ & $\%$ & $n$ & $\%$ & \\
\hline Sim & 5 & 45,45 & 6 & 54,55 & 11 \\
\hline Não & 1 & 4,17 & 23 & 95,83 & 24 \\
\hline TOTAL & 6 & & 29 & & 35 \\
\hline
\end{tabular}

Teste Exato de Fisher $(\mathrm{p}=0,0071)$

n- número absoluto

Tabela 4 - Distribuição dos pacientes com hipertensão arterial, quanto ao uso de medicamentos para pressão arterial

\begin{tabular}{lc}
\hline $\begin{array}{l}\text { Medicamentos } \\
\text { hipertensão }\end{array}$ & $\mathrm{n}(\%)$ \\
\hline Sim & $45(97,9 \%)$ \\
Não & $1(2,1 \%)$ \\
\hline Total & $46(100 \%)$ \\
\hline n- número absoluto &
\end{tabular}




\section{DISCUSSÃO}

Neste estudo verificou-se uma grande freqüência de queixa de vertigem em pacientes com hipertensão arterial entre 45 e 64 anos e sem perda auditiva. Uma vez que foi encontrada associação significante entre a queixa de vertigem e hipertensão arterial nesta população, sugere-se que este sintoma deve ser investigado e tratado na população de hipertensos, com intuito da melhoria da qualidade de vida.

Na presente pesquisa houve o cuidado em limitar a idade dos sujeitos, concentrando a faixa etária dos indivíduos de meia idade, entre 45 e 64 anos como em outras pesquisas realizadas na área de hipertensão ${ }_{11,12}$. Os critérios de exclusão, eliminando aqueles indivíduos com enfermidades, capazes de produzir vertigem e os cuidados na constatação da queixa de vertigem e na verificação da hipertensão arterial serviram certamente, para reduzir os vieses de seleção. Porém, durante a anamnese, os vieses repetitivos de memória, quanto ao uso de medicamentos ototóxicos, podem ter afetado os resultados, embora as informações sobre o uso de medicamentos utilizados pelos indivíduos mostrem medidas elevadas de confiabilidade ${ }^{13}$, mesmo na presença de fatores confundíveis de informação, como por exemplo, a respeito de alterações metabólicas e vasculares ainda não diagnosticadas ou não relatadas pelo paciente.

O número de doenças crônicas aumenta com o passar dos anos, sendo em idosos grande a incidência tanto de hipertensão arterial sistêmica como de vertigem $^{2,14}$. Alguns estudos justificam que a vertigem ocorrida com o passar da idade tem relação com uma insuficiência microcirculatória decorrente de uma oclusão vascular por embolia, hemorragia ou vasoespasmo e que estes por sua vez, seriam decorrentes de uma síndrome de hiperviscosidade ou microangiopatia por diabetes ou hipertensão, sendo que a hipertensão poderia, por meio desses fatores histopatológicos, provocar alterações no ouvido interno ${ }^{15,16}$. Isto poderia justificar os resultados encontrados nesta pesquisa.

No entanto a exposição a determinadas substancias e alterações sistêmicas ou determinados estilos de vida, também podem acelerar o processo de degeneração do aparelho auditivo, tais como, inalação de substâncias tóxicas, certas alterações circulatórias e metabólicas além de infecções, traumas de várias naturezas e hereditariedade ${ }^{17-20}$. Neste trabalho, com intuito de reduzir problemas de vício de seleção, foram excluídos indivíduos com diabetes, com história de internações e ingestão de medicamentos ototóxicos.

No que se refere ao gênero, ocorreu uma diferença no número de indivíduos do gênero feminino e masculino devido os sujeitos terem sido alocados na seqüência dos atendimentos da clínica.
De acordo com o último Censo realizado no Brasil em 2000, a população idosa correspondia a $5,85 \%$ da população, sendo o crescimento de $1,02 \%$ em relação ao Censo anterior na década de 90. O índice de envelhecimento também aumentou de 13,90\% em 1991 para 19,77\% em $2000{ }^{14}$. Isto vem ocorrendo à diminuição das taxas de mortalidade e conseqüente elevação da esperança média de vida no mundo inteiro, pois em virtude dos progressos da medicina aliados aos fatores sócio-econômicos vêm diminuindo consideravelmente o número de mortes prematuras e com isso as doenças cardiovasculares e o câncer estão sendo as principais causas de morte da atualidade. Isto se deve não ao aumento da incidência destas patologias, mas à maior longevidade e principalmente, ao controle e eficácia dos medicamentos na eliminação das doenças infecciosas ${ }^{9,21}$.

As doenças crônicas determinadas por problemas genéticos, estilo de vida, meio ambiente e ao próprio envelhecimento passam então a serem os principais problemas de saúde do idoso, sendo necessário a partir daí atenção especial aos idosos com objetivo de aumentar o número de indivíduos saudáveis e independentes, capazes de manter ao máximo as funções físicas e mentais, com atenção especial aos portadores de alterações sistêmicas como hipertensão, diabetes mellitus e alterações hormonais ${ }^{9,20-23}$. Chama-se isso de "compressão da morbidade", que significa viver uma vida relativamente saudável e comprimir as doenças em um curto período de tempo o mais próximo da morte ${ }^{24}$.

É preciso então que sejam inseridos princípios e estratégias de cuidados preventivos e manutenção da saúde que deverão ser dirigidas particularmente a cada paciente visando maior qualidade de vida. Entre estes cuidados preventivos estão os cuidados com os inúmeros malefícios provenientes da hipertensão arterial, entre os quais, a perda auditiva e a vertigem ${ }^{8,25}$.

As atitudes tomadas certamente ajudarão os muitos pacientes que pela tontura e mais especificamente as vertigens, deliberadamente restringem as atividades físicas, viagens e reuniões sociais, com o intuito de reduzir o risco de aparecimento destes sintomas desagradáveis e assustadores, e para evitar o embaraço social e o estigma que eles podem causar ${ }^{25}$.

\section{CONCLUSÃO}

Neste estudo verificou-se uma relação significante de queixa de vertigem em pacientes com hipertensão arterial entre 45 e 64 anos e sem perda auditiva, o que sugere que este sintoma deva ser investigado e tratado na população de hipertensos, com intuito da melhoria da qualidade de vida. 


\section{ABSTRACT}

Purpose: to investigate the presence of vertigo complaint in middle-aged hypertension patients. Methods: a transversal study. Composed by 154 patients of both genders, aged from 45 to 64 years, included in the research after sample estimation. Hypertension was verified through blood pressure readings and by a systematized questionnaire about hypertension and the use of medication for blood pressure. Vertigo was assessed through audiological anamneses. Results: there is a significant association between blood hypertension and vertigo. Conclusion: the results in this research, through evidence of association between blood hypertension and vertigo complaint, can be a base for health professionals concerned with alterations caused by blood hypertension.

KEYWORDS: Hypertension; Vertigo; Dizziness; Quality of Life; Middle Aged

\section{REFERÊNCIAS}

1.Dix MR, Hood JD. Vertigo. Chichester: Wiley; 1984. $235 \mathrm{p}$.

2.Brant T. Vertigo: its multisensory syndromes. Londres: Springer-Verlag; 1990. 256 p.

3. Konrad HR, Girardi M, Helfert R. Balance and aging. Laryngoscope. 1999; 109(9):1454-60.

4.Mor R, Fragoso M, Figueiredo JF, Taguchi CK. Vestibulometria e fonoaudiologia. São Paulo: Lovise; 2001.

5.Katz J. Tratado de audiologia clínica. São Paulo: Manole; 1989.

6.Marková M. The cochleovestibular syndrome in hypertension. Cesk Otolaryngol. 1990; 39(2):89-97.

7.Bachor E, Selig YK, Jahnke K, Rettinger G, Karmody CS. Vascular variations of the inner ear. Acta Otolaryngol. 2001; 121(1):35-41.

8. Marchiori LLM, Rego Filho EA, Matsuo T. Hipertensão como fator associado à perda auditiva. Rev Bras Otorrinolaringol. 2006; 72(4):533-40.

9.III Consenso Brasileiro de Hipertensão Arterial. Campos do Jordão, SP. 12 a 15 de Fevereiro de 1998.

10. European Society of Hypertension - European Society of Cardiology Guidelines Commitee. 2003 European Society of Hypertension - European Society of Cardiology Guidelines for the management of arterial hypertension. J Hypertens. 2003; 21(6):1011-53. 11. Antikainen R, Jousilahti P, Tuomilehto J. Systolic blood pressure, isolated systolic hypertension and risk of coronary heart disease, strokes, cardiovascular disease and all-cause mortality in the middle-aged population. J Hypertens. 1998; 16(5):577-83.

12. Antikainen $R L$, Jousilahti $P$, Vanhanen $H$, Tuomilehto J. Excess mortality associated with increased pulse pressure among middle-aged men and women is explained by high systolic blood pressure. J Hypertens. 2000; 18(4):417-23.
13. Coutinho ESF, Silva SD, Paiva CHA, Freitas GG, Miranda LM, Mendonça R. Confiabilidade da informação sobre o uso recente de medicamentos em um estudo caso-controle de base hospitalar. Cad Saúde Públ. 1999; 15:553-8.

14. Baraldi GS, Almeida LC, Borgea ACLC. Hearing loss and hypertension: findings in an older by group. Rev Bras Otorrinolaringol. 2004; 70(5):640-4.

15. Otaviani F. Presbycusis. Acta Otorhinolaryngol Ital. 1998; 18(4 Suppl 59):74-7.

16. Carrasco VN, Prazma J, Faber JE, Triana RJ, Pillsbury HC. Cochlear microcirculation: effect of adrenergic agonists on arteriole diameter. Arch Otolaryngol Head Neck Surg. 1990; 116(4):411-7.

17. ASHA (American Speech, Language and Hearing Association). Disponível em: URL: www.asha.org . Acesso em: 29/10/2004.

18. Marchiori LLM, Gibrin PCD. Diabetes mellitus: prevalence of hearing disorders. Arq Bras Endocrinol Metab. 2003; 47(1):82-6.

19. Collet L, Berger-Vachon C, Desreux V, Morgon A. Auditory brainstem response (ABR) latency: relative importance of age, sex and sensorineural hearingloss using a mathematical model of the audiogram. Int J Neurosci. 1992; 67(1-4):187-97.

20. Marchiori LLM, Freitas SV, Vieira M. Análise de prevalência das queixas e perdas auditivas de pacientes com hipertensão arterial submetidos à avaliação audiológica. Fono Atual. 2002; 21 (2):97-104.

21. Datasus 2004. Dados de morbidade hospitalar e mortalidade. Disponível em: URL: <http:// www.datasus.gov.br> Acesso em: 20/04/2004.

22. Ferreira DR, Silva AA. Aging and life quality: an otorhinolaryngological review. Rev Laryngol Otol Rhinol. 2004; 125(3):143-50.

23. Kornet L, Hoeks AP, Janssen BJ, Houben AJ, De Leeuw PW, Reneman RS. Neural activity of the cardiac baroreflex decreases with age in normotensive 
and hypertensive subjects. J Hypertens. 2005; 23(4):815-23.

24. Fries JF. Aging, natural death and the compression of morbidity. N Engl J Med. 1980; 303(3):130-5.
25. Ganança FF, Castro ASO, Branco FC, Natour J. Interferência da tontura na qualidade de vida de pacientes com síndrome vestibular periférica. Rev Bras Otorrinolaringol. 2004; 70(1):97-104.

RECEBIDO EM: 30/11/06

ACEITO EM: 22/02/07

Endereço para correspondência:

Rua Belo Horizonte 1399 / 401

Londrina - PR

CEP: $86020-060$

Tel: (43) 33236068

E-mail: luciana.marchiori@.unopar.br 